\title{
Pyoderma gangrenosum as the first clinical manifestation of primary Sjögren disease: A case report and review of literature
}

\author{
A Malek ${ }^{1 *}, \mathrm{~J} \mathrm{Saliba}^{2}, \mathrm{~J} \mathrm{Choucair}^{1}$ and C Irani ${ }^{2}$ \\ ${ }^{1}$ Infectious diseases, at Saint Joseph University, Faculty of Medicine/Hotel Dieu De France Hospital, Beyrouth, Lebanon \\ ${ }^{2}$ Internal medicine, at Saint Joseph University, Faculty of Medicine/Hotel Dieu De France Hospital, Beyrouth, Lebanon
}

\begin{abstract}
Introduction: Pyoderma gangrenosum (PG) is a rare neutrophilic dermatosis characterized by painful cutaneous ulcers. It can appear as primary or idiopathic illness or secondary to a systemic disease. Only few cases in the literature reported the association between PG and Sjögren disease, which manifests primarily with sicca symptoms. This paper describes the remarkable initial clinical presentation of primary Sjögren disease revealed by diffuse and severe PG that improved dramatically after treatment initiation.

History: A 25-year-old female, with previous history of ocular dryness, was hospitalized after the rapid progression of severe skin eruption which first started at the lower limbs, then affected her face and trunk. The transformation of the cutaneous rash from non-pruritic papules into extremely painful, well defined, hemorrhagic bullae with central scarring was clinically consistent with PG. Pathergy sign was present. Skin biopsy showed an aspect of neutrophilic dermatosis. ANA profile revealed positive anti-SSA/Ro and positive anti-SSB/La. Since the patient also had xerophthalmia, xerostomia and highly positive Schirmer's test, she was diagnosed with PG secondary to Sjögren's syndrome.
\end{abstract}

She responded well to systemic steroids tapered to the oral form then oral cyclosporine along with local wound care.

Conclusion: This is the first case report that illustrates severe PG as the first clinical manifestation of Sjögren's syndrome, which has to be ruled out in the setting of each neutrophilic dermatosis, and can be successfully treated with corticosteroids and cyclosporine.

\section{Introduction}

Pyoderma gangrenosum (PG) is a rare neutrophilic dermatosis, presented by painful ulcerative skin lesions that can occur at any age, but most commonly in young to middle-aged adults. It affects women more frequently than men. Pyoderma gangrenosum often begins as a small papule or pustule that progressively expands and ulcerates, with an acneiform and serpiginous necrotizing red-blue border [1-3]. This painful lesion is usually located over the lower extremities [4], but it can involve any cutaneous site (the scalp, face, periocular and perioral areas, arms, hands, trunk, genital, perianal, and peristomal). Furthermore, pyodermic lesions could be triggered in $30 \%$ of cases by minor trauma (pathergy) [1,2]. PG could be primary or secondary to multiple systemic diseases such as inflammatory bowel diseases being the most common, then hematologic malignancies and rheumatologic disorders [2].

The pathogenesis of pyoderma gangrenosum is not well understood. It seems to be a multifactorial combination of genetic predisposition, neutrophil dysfunction and inflammatory mediators [5]. The diagnosis of pyoderma gangrenosum is mainly clinical but the importance of laboratory tests and the histopathological exam is to detect any associated diseases or to exclude other diseases that could mimic PG, such as malignancies, vascular disorders, and infections. The clinical course could be variable, ranging from rapid evolving disease to more indolent forms.
Sjögren's syndrome (SS) is a systemic autoimmune disease characterized by lymphocytes infiltration of exocrine glands, especially the lacrimal and salivary glands. The inflammation process damages and destroys the glands, leading to dry eyes and mouth, also referred to xerophthalmia and xerostomia respectively [6,7]. Sjögren's syndrome may occur as a primary disease, or secondary to another autoimmune connective tissue disorder or systemic conditions. Women are mostly affected and the usual onset is in middle age. Nowadays, there is no curative treatment and management is usually given for symptoms relief and prevention of complications including opportunistic infections. Follow-up is necessary to monitor the increased risk of hematologic malignancies and inflammatory-associated disorders of other vital organs such as lungs, liver, skin, kidneys, pericardium and central nervous system [7].

Although, Sjögren's syndrome manifests primarily as sicca symptoms, it is a heterogeneous process that can include a wide spectrum

Correspondence to: Alexandre Malek MD, Infectious diseases, at Saint Joseph University, Faculty Of Medicine/Hotel Dieu De France Hospital, Beyrouth, Lebanon, E-mail: alexandermalek@hotmail.com

Key words: pyoderma gangrenosum, neutrophilic dermatosis, Sjögren disease, internal disease associated with dermatology

Received: October 23, 2017; Accepted: November 15, 2017; Published: November 18, 2017 
of extraglandular clinical presentations, involving gastrointestinal, pulmonary, neurologic, articular, and cutaneous organs [8]. The skin involvement in primary Sjögren's syndrome is displayed in at least half of the patients; and lesions can be vascultitic or non-vasculitic in origin [9]. The skin manifestations are diverse ranging from xeroderma being the most frequent and benign to more severely pronounced lesions that worsen prognosis such as palpable, non-palpable purpura and urticarial vasculitis [8].

Only few cases in the literature have described the unusual association between pyoderma gangrenosum and Sjögren's syndrome. In this paper, we describe the remarkable initial clinical presentation of primary Sjögren disease revealed by diffuse and severe pyoderma gangrenosum. In addition, the particularity of this case resides in the spectacular and dramatic improvement after treatment initiation.

\section{Case presentation}

We report the case of a 25 -year-old female, only known to have ocular dryness treated with artificial tears, with no history of drug or food allergy. She presented to the office for throat pain and lowgrade fever, treated as bacterial pharyngitis with amoxicillin-clavulanic acid for ten days of duration. Four days later, she had noticed a skin eruption, which started mainly over her lower limbs bilaterally (Figures 1-3), and then the lesions evolved to affect her upper lip (Figure 4) and back (Figure 5). The cutaneous rash began with non-pruriginous papules that rapidly transformed into extremely painful hemorrhagic bullae with central scarring and well-defined borders, leading to the patient hospitalization. By reviewing history, she has been complaining of dry mouth. No weight loss, night sweats, conjunctivitis, neither mucosal lesions nor aphtosis. She denied blurry vision, shortness of

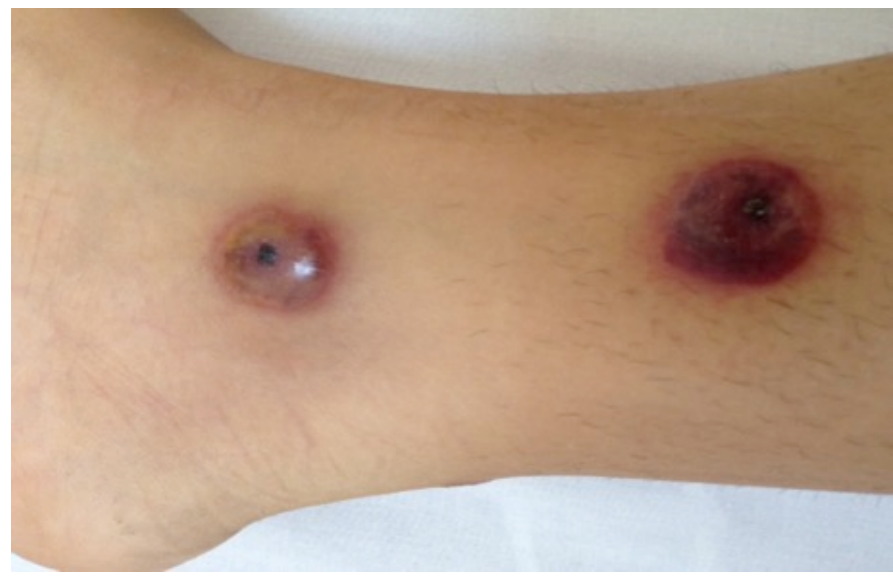

Figure 1. Painful hemorrhagic bullae with central scarring

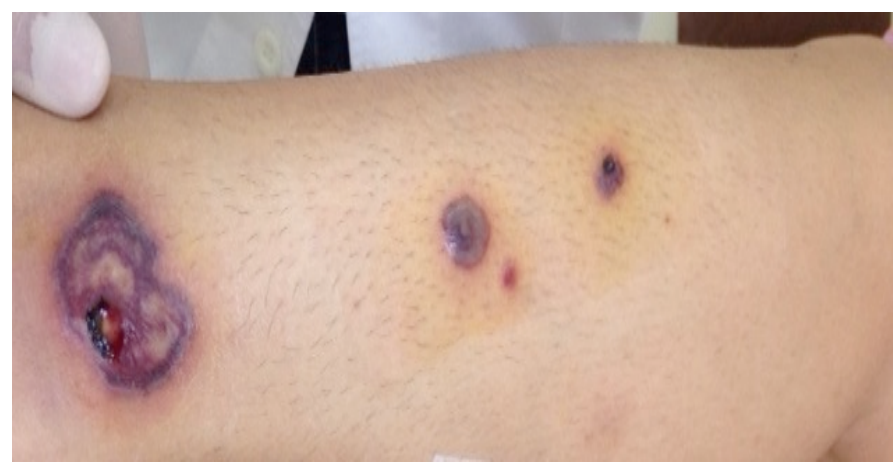

Figure 2. Left external malleolus ulceration with serpiginous necrotizing red-blue border

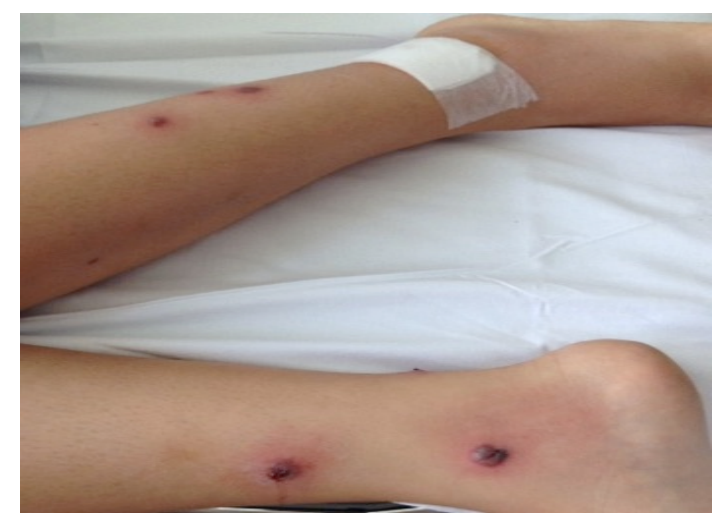

Figure 3. Bilateral lower limbs hemorrhagic bullae skin lesions

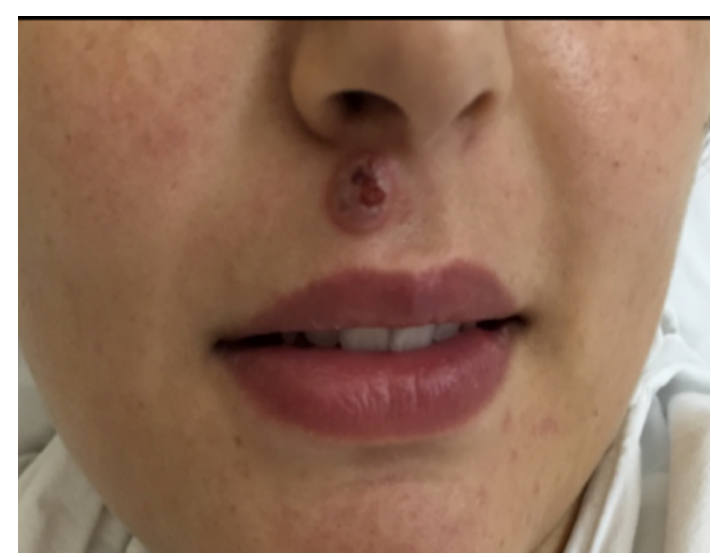

Figure 4. Upper lip lesion with central necrosis

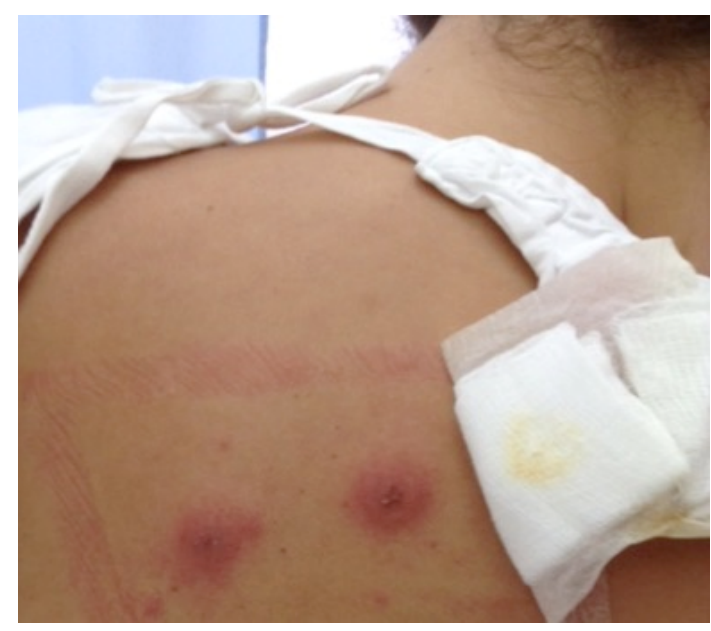

Figure 5. Skin lesions with central ulceration on the back

breath, abdominal pain, arthralgia, and changes in bowel habits. There were no epistaxis, melena, hematochezia or hematuria.

She admitted occasional smoking, but no alcohol intake or illicit drugs use. Her family history was remarkable for Hashimoto disease and rheumatoid arthritis.

On physical exam, she was conscious, cooperate, oriented, but uncomfortable due to the excruciating pain of her skin lesions. No ecchymosis or lymphadenopathy was detected. The cardiopulmonary examination was normal, and the abdominal examination revealed no abdominal tenderness or hepato-splenomegaly. Musculo-skeletal exam was normal. 
The on-admission laboratory blood exam showed: hemoglobin $11.4 \mathrm{~g} / \mathrm{dL}$, hematocrit $35.2 \%$, WBC $9,100 / \mathrm{mm}^{3}$, $83 \%$ neutrophils, $1 \%$ eosinophiles, $10 \%$ lymphocytes, CRP $35.3 \mathrm{mg} / \mathrm{L}$, creatinine $0.55 \mathrm{mg} /$ dl, BUN $10.36 \mathrm{mg} / \mathrm{dl}$, LDH $640 \mathrm{U} / \mathrm{L}$, bilirubin $0.87 \mathrm{mg} / \mathrm{dl}$, direct bilirubin $0 \mathrm{mg} / \mathrm{dl}$, gamma glutamine transferase $25 \mathrm{U} / \mathrm{L}$, AST $18 \mathrm{U} / \mathrm{L}$, ALT $13 \mathrm{U} / \mathrm{L}$, alcaline phosphatase $86 \mathrm{U} / \mathrm{L}$. HIV, HBV, HCV serologic tests and PDD skin test were negative. Serologic tests for Chlamydia (pneumoniae and trachomatis), Mycoplasma pneumoniae and Herpes Simplex virus were negative. Serum protein electrophoresis and immunoelectrophoresis showed the following: Total proteins $7.8 \mathrm{~g} / \mathrm{dl}$, albumin $3.46 \mathrm{~g} / \mathrm{dl}$, alpha $10.25 \mathrm{~g} / \mathrm{dl}$, alpha $21.01 \mathrm{~g} / \mathrm{dl}$, Beta $1.15 \mathrm{~g} / \mathrm{d}$, gamma 1.93 g/dl, total IgA 2.63 g/l, IgG 19.3 g/l, IgM 1.58 g/l, Kappa chains $4.35 \mathrm{~g} / \mathrm{l}$, and Lambda chains $2.94 \mathrm{~g} / \mathrm{l}$. Culture taken from the wound surface was sterile. Antinuclear antibody (ANA) turned out to be positive at a titer of 1/640, homogenous form. C-ANCA, p-ANCA, and double stranded DNA were negative. The ANA profile done with the Enzyme Linked ImmunoSorbant Assay (ELISA) showed positive anti-SSA/Ro: 6.5 (Normal <1), and positive anti-SSB/La: 2 (Normal $<1)$. Those autoantibodies, which target ribonucleoproteins, are most commonly associated with Sjögren's syndrome and with higher incidence of extra glandular manifestations.

Although anti-Ro/SSA antibodies can be present in over $70 \%$ of patients with SS, they are not specific for this disease as they can also be found in SLE and other autoimmune diseases even in the absence of sicca signs and symptoms. On the other hand, anti-La/SSB is more specific; and it is found in $50 \%$ of patients with primary SS or SS/SLE [10]. The simultaneous positivity for anti-Ro/SSa and anti-Ro/SSB is more specific for SS than for SLE [11]. Moreover, IgG polyclonal gammopathy could be present in the context of a non-specific systemic inflammatory reaction that occurs in Sjögren's syndrome.

Skin biopsy was taken urgently. Antibiotics were withdrawn due to the suspicion of a type III hypersensitivity reaction secondary to betalactam antibiotics intake. Corticosteroids were initiated at a dose of 80 mg of IV solumedrol once daily directly after the biopsy and colchicine $1 \mathrm{mg}$ per day. During hospitalization, the patient developed a small pustule over the insertion site of the peripheral catheter, suggestive of Pathergy sign (Figure 6). Pathergy sign is a nonspecific inflammatory skin reaction that occurs in response to minimal trauma or intradermal saline injection, seen mainly in PG and Behcet disease [7]. Due to the absence of oral or genital aphtosis, vascular and ocular lesions, Behçet disease was unlikely. Negative HLA B51 with positive HLA B52 complies with our low clinical suspicion.

The result of skin biopsy showed an aspect of neutrophilic dermatosis compatible with pyoderma gangrenosum, with close secondary arteriolar and venular alterations. Immunofluorescence was negative. Some granulomatous formations were present. Those results in addition to the typical skin manifestations and evolution including pathergy sign were compatible with PG.

Gastroscopy and colonoscopy were done to rule out inflammatory bowel disease and came normal. Thoraco-abdominopelvic CT scan effectuated to eliminate any underlying malignancies such as lymphoma was negative.

She was on Solumedrol $80 \mathrm{mg}$ IV once daily with a good initial response and she was discharged on oral prednisone $60 \mathrm{mg}$ once daily plus colchicine $1 \mathrm{mg}$ daily and wound care. Three weeks later, we hospitalized the patient because she noticed a necrosis of the lesions in the lower limbs only (Figure 7) and aggravation of pain. However, there is a remarkable improvement of other skin lesions described

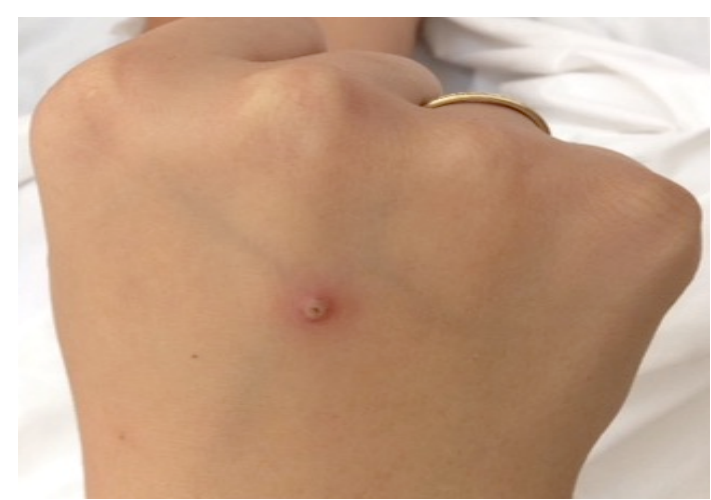

Figure 6. Pathergy sign at the insertion site of peripheral catheter

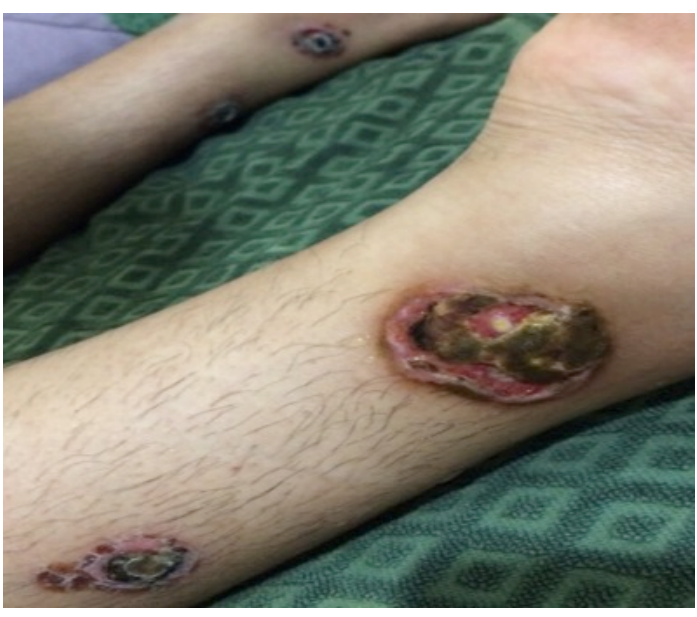

Figure 7. Skin necrosis

above. We initiated solumedrol $80 \mathrm{mg}$ IV once daily and we added cyclosporine $100 \mathrm{mg}$ twice daily along with pain medications and wound debridement (Figure 8).

According to the patient symptoms: xerophthalmia and xerostomia, we did Schirmer's test and it was highly positive which confirmed the diagnosis of Sjögren's syndrome. Accessory salivary gland biopsy was performed and the result revealed a minimal salivary gland inflammation.

Fortunately, she responded well to systemic followed by oral corticosteroid $60 \mathrm{mg}$ along with cyclosporine $100 \mathrm{mg}$ twice daily and wound dressings. On three months follow-up after the first hospitalization; she had a great improvement of skin lesions (Figure 9) along with significant control of symptoms of Sjögren's syndrome.

\section{Discussion}

In the last few years, all the published articles and studies showed that pyoderma gangrenosum (PG) is not merely an isolated skin condition but instead it is a cutaneous manifestation of a generalized autoimmune and inflammatory reaction related with other systemic diseases. PG is considered part of the spectrum of the neutrophilic dermatoses (NDs) entity characterized by skin lesions for which immunohistological examination reveals important inflammatory reaction composed primarily of neutrophils, with no evidence of infection. Typical NDs include pyoderma gangrenosum, sweet syndrome, sub-corneal pustular dermatosis, and erythema elevatum diutinum. Until now, the pathophysiology of NDs is not well known, 


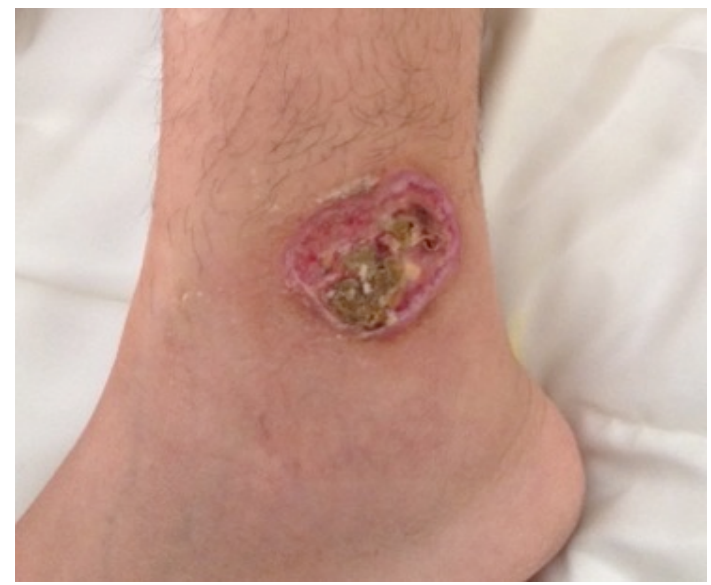

Figure 8. After skin debridement

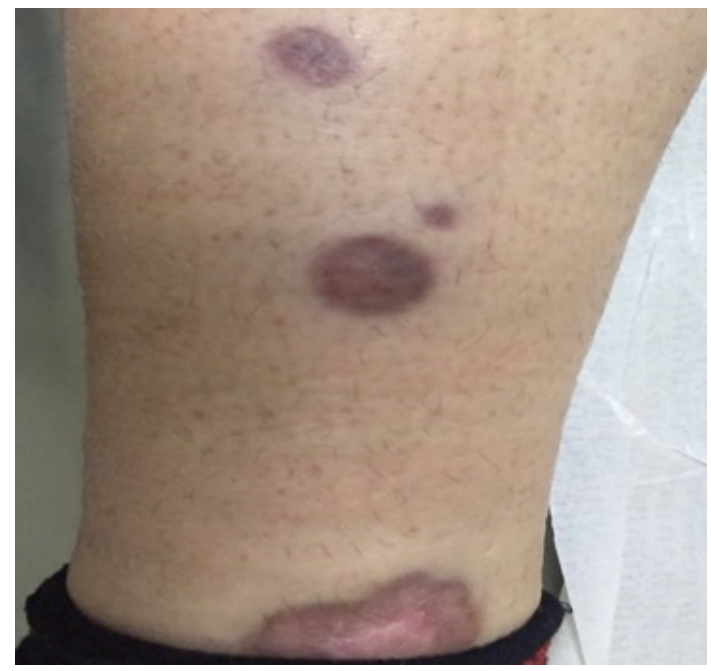

Figure 9. At three months follow-up

but recent research suggests that they should be classified within the spectrum of "polygenic" autoinflammatory diseases [12]. Neutrophilic dermatoses may be associated with multiple systemic disorders, such as myeloproliferative disorders, monoclonal gammopathies (mainly IgA type), inflammatory bowel diseases, and autoimmune connective tissue diseases (AICTDs). AICTDs include lupus erythematosus, dermatomyositis, Sjögren's syndrome, rheumatoid arthritis, and systemic sclerosis [12].

Sjögren's syndrome (SS) is a multisystem autoimmune disease and many organs other than the exocrine glands may be affected such as the lungs, heart, and gastrointestinal tract, including the pancreas and liver; the skin and joints; the kidneys, bladder, and gynecologic system; and both the peripheral nervous system (PNS) and central nervous system (CNS). In addition, hematologic abnormalities are often present and the risk of lymphoproliferative disorders is increased. Cutaneous vasculitis and Raynaud phenomenon may occur. Based on the revised American-European Consensus Group (AECG) criteria, which are the gold standard for making the diagnosis, our patient fulfilled the criteria: she has ocular and oral dryness, a positive Schirmer's test and anti-SSA/ SSB autoantibodies. Lip biopsy showed minimal accessory salivary glands inflammation, knowing that the biopsy was done after one month of treatment.
Only rare cases of primary Sjögren's syndrome and pyoderma gangrenosum have been reported in the literature. Ravic-Nicolic et al. [13] reported the first case of a 61 year-old patient with a two-year history of Sjögren's syndrome diagnosed with pyoderma gangrenosum on the posterior side of the left thigh based on clinical grounds. Another case report by Yi-Hsuan Tsai et al. [14] described a 52 year-old woman with intractable leg ulcers diagnosed as pyoderma gangrenosum based on skin biopsy, and later Sjögren Syndrome was confirmed by the presence of xerostomia, xerophtalmia, and by immunologic study along with positive Schirmer's test. Gail et al. [15] described a case of 46 year-old African American female patient with rheumatoid arthritis and Sjögren syndrome that developed a painful skin ulceration on the right internal malleolus diagnosed as pyoderma gangrenosum based on clinical appearance with the absence of other etiologies on skin biopsy. Badea et al. [16] reported the case of a 61 year-old Caucasian female patient known to have Sjögren syndrome for the last 10 years who presented with painful cutaneous ulcerations over the right shin. SS was controlled on Aziathioprine $20 \mathrm{mg}$ /day plus prednisone $5 \mathrm{mg} /$ day, until the patient stopped the treatment two months before admission. They established the diagnosis of pyoderma gangrenosum on the background of a primary Sjögren Syndrome based on clinical presentation, immunologic studies, and skin biopsy. Anderson et al. [17] described the case of a 94 year-old male with primary Sjögren syndrome who presented with painful leg ulcers developing within 2-week duration in previously normal skin that was diagnosed as pyoderma gangrenosum.

In all the cases mentioned above, pyoderma gangrenosum was diagnosed in patients who were already known to have Sjögren's syndrome. To the best of our knowledge, the importance of our case lies in the fact that it is the only reported case of pyoderma gangrenosum as the first clinical manifestation of Sjögren's syndrome [18].

\section{Conclusion}

The association of pyoderma gangrenosum and Sjögren's syndrome is extremely rare, and this is the first case reported in literature of PG as initial presentation of SS. And despite the fact that PG is an unusual presentation, this case provides a clue to clinicians that SS might be considered as a cause of pyoderma gangrenosum. Interestingly, the combination of corticosteroids and cyclosporine are an effective and predictable treatment of pyoderma gangrenosum in-patient with Sjögren's syndrome.

\section{References}

1. Al Ghazal P, Herberger K, Schaller J, Strölin A, Hoff NP, et al. (2013) Associated factors and comorbidities in patients with pyoderma gangrenosum in Germany: a retrospective multicentric analysis in 259 patients. Orphanet J Rare Dis 8: 136

2. Cozzani E, Gasparini G, Parodi A (2014) Pyoderma Gangrenosum: a systemic review. G Ital Dermatol Venereol 149: 587-600. [Crossref]

3. Bhat RM (2012) Pyoderma gangrenosum: An update. Indian Dermatol Online J 3 : 7-13. [CrossRef]

4. Powell FC, Schroeter AL, Su WP, Perry HO (1985) Pyoderma gangrenosum: a review of 86 patients. Q J Med 55: 173-186. [CrossRef]

5. Braswell SF, Kostopoulos TC, Ortega-Loayza AG (2015) Pathophysiology of pyoderma gangrenosum (PG): An updated review. J Am Acad Dermatol 73: 691-698. [Crossref]

6. von den Driesch P (1997) Pyoderma gangrenosum: a report of 44 cases with follow-up. Br J Dermatol 137: 1000-1005. [CrossRef]

7. Kruszka P, O'Brian RJ (2009) Diagnosis and management of Sjögren syndrome. $A m$ Fam Physician 79: 465-470. [CrossRef]

8. Kittridge A, Routhouska SB, Korman NJ (2011) Dermatologic manifestations of Sjögren syndrome. J Cutan Med Surg 15: 8-14. [Crossref] 
9. Soy M, Piskin S (2007) Cutaneous findings in patients with primary Sjogren's syndrome. Clin Rheumatol 26: 1350-1352. [Crossref]

10. Mavragani CP, Tzioufas AG, Moutsopoulos HM (2000) Sjögren's syndrome: autoantibodies to cellular antigens. Clinical and molecular aspects. Int Arch Allergy Immunol 123: 46-57. [Crossref]

11. Yang Z, Liang Y, Zhong R (2012) Is Identification of Anti-SSA and/or-SSB Antibodies Necessary in Serum Samples Referred for Antinuclear Antibodies Testing? J Clin Lab Anal 26: 447-451. [Crossref]

12. Hau E, Vignon Pennamen MD, Battistella M, Saussine A, Bergis M, et al. (2014) Neutrophilic skin lesions in autoimmune connective tissue diseases: nine cases and a literature review. Medicine (Baltimore) 93: e346. [Crossref]

13. Ravic-Nikolic A, Milicic V, Ristic G, Jovovic-Dagovic B (2009) Pyoderma gangrenosum associated with Sjögren syndrome. Eur J Dermatol 19: 392-393. [Crossref]
14. Tsai YH, Huang CT, Chai CY, Wu CC (2014) Pyoderma gangrenosum: Intractable leg ulcers in Sjogren's syndrome. Kaohsiung J Med Sci 30: 486-488. [Crossref]

15. Kerr GS, Aggarwal A, McDonald-Pinkett S (2012) A woman with rheumatoid arthritis, Sjögren's Syndrome, leg ulcer, and significant weight loss. Arthritis Care Res (Hoboken) 64: 785-792. [Crossref]

16. Badea MA, Tilea AM, Cotoi OS, Vartolomei MD, Hodasz I, et al. (2014) Pyoderma gangrenosum in a patient with primary sjögren's syndrome-case report. Bulletin of the Transilvania University of Brasov 7.

17. Anderson ML1, Mackenzie G (2014) When an ulcer is not 'just an ulcer': pyoderma gangrenosum. BMJ Case Rep bcr2013203445. [Crossref]

18. Scherrer MA, de Castro LP, Rocha VB, Pacheco L (2014) The dermatoscopy in the skin pathergy testing: case series in patients with suspected Behçet's Disease. Rev Bras Reumatol 54: 494-498. [Crossref]

Copyright: (C2017 Malek A. This is an open-access article distributed under the terms of the Creative Commons Attribution License, which permits unrestricted use, distribution, and reproduction in any medium, provided the original author and source are credited. 